\title{
Influence de l'âge des repousses sur la dégradabilité de l'azote d'une végétation naturelle de savane en région de climat tropical humide après correction de la contamination azotée bactérienne
}

\author{
P Lecomte 1, S Chabi 2, Y Beckers 2, M Khamoun 2, A Théwis 2 \\ 'CRAGx, Station de Haute Belgique, 6800 Libramont ; "FSAGx, UER Zootechnie, 5030 Gembloux, Belgique
}

L'étude visait à caractériser sur des repousses de différents âges les cinétiques de dégradation ruminale pour établir la valeur de dégradabilité théorique de la matière protéique. Les échantillons prélevés au Togo (Ranch de l'Adélé), concernent 4 stades de repousse après feux et ont été collectés en début de saison des pluies. Ils sont constitués d'une part importante d'Hyparhenia smithiana et de Loudetia arundinacea. L'âge du fourrage, estimé en se référant à la date du feu organisé dans la parcelle, était respectivement pour chacun des quatre échantillons de 15, 25, 45 et 65 jours. Présèchés au soleil et ensuite étuvés $\left(60^{\circ} \mathrm{C}\right.$ pendant $\left.36 \mathrm{~h}\right)$, les échantillons ont été broyés à la grille de $1 \mathrm{~mm}$. La dégradabilité des constituants a été étudiée par la technique des sachets.

Nous avons utilisé trois boeufs (race Pie Noire), équipés chacun d'une fistule du rumen. La ration de base distribuée à raison de $10 \mathrm{~kg}$ de MS par jour, était constituée de $80 \%$ de foin de prairie et $20 \%$ de concentrés.

Trois périodes de mesure ont permis de tester les quatre aliments sur les trois animaux avec des temps de séjours de $0,4,8,16,48,72,96$, 120 heures. Après lavage, séchage et établissement du \% de matière sèche disparue, les sachets ont été regroupés par temps d'incubation de façon à disposer de quantités suffisantes pour effectuer une mesure en spectrométrie proche infra rouge et l'analyse Kjeldahl de la teneur en protéine du résidu. Pour estimer la contamination azotée bactérienne sur les résidus des 4 échantillons

\begin{tabular}{lc} 
Age des repousses (jours) & 15 \\
\hline MAT (\% MS) & 11,7 \\
N-NDF (\% MAT) & 8,93 \\
N-ADF $\%$ MAT) & 1,77 \\
N-ADL (\% MAT) & 1,66 \\
DT MAT corrigée & 53,5 \\
$\triangle$ non corrigé & 13,2
\end{tabular}

nous avons fait appel à une prédiction en spectrométrie proche infrarouge (Lecomte et al, 1994, Proc Soc Nutr Physiol, 3, 44). Les cinétiques de dégradation ont été ajustées selon le modèle de Ørskov et Mac Donald (1979, J Agric Sci Camb, 92, 449-503). Pour le calcul de la dégradabilité théorique (DT) le paramètre $\mathrm{k}$ a été fixé à une valeur de $6 \%$.

Corrigées de la contamination bactérienne les valeurs de dégradabilité théorique de la protéine sont réévaluées de 15 à 19 points selon l'âge du fourrage. Les valeurs de DT sont en moyenne de $53 \%$ pour les fourrages les plus jeunes, elles décroissent ensuite et n'atteignent plus que $39 \%$ pour le fourrage le plus âgé caractérisé par une teneur en azote moindre et une part plus importante de l'azote lié aux fibres. Le biais dû à la contamination croît jusqu'à l'âge de 45 jours il décroît légèrement à 65 jours du fait d'une contamination apparemment plus lente, en valeur prédite, du fourrage le plus âgé. A défaut d'être une mesure exacte de la contamination, la spectrométrie infra rouge a permis une estimation rapide et peu coûteuse de la part d'azote microbien et la correction de la DT. La valeur DT $=53 \%$ proposée par Xandé (1992, Valeurs alimentaires des fourrages de la zone tropicale humide, ed. INRA) serait ainsi confirmée pour le fourrage jeune (2 à 4 semaines de repousse) ; elle apparaît surestimée pour des repousses plus âgées moins riches en azote et à teneur en fibres plus élevée.

$\begin{array}{ccc}25 & 45 & 65 \\ 9,2 & 7,4 & 6,0 \\ 9,17 & 10,43 & 10,39 \\ 1,91 & 2,58 & 2,41 \\ 1,67 & 1,66 & 2,35 \\ 53,9 & 48,8 & 39,4 \\ 15,5 & 19,4 & 17,0\end{array}$

$\Delta=$ DTc - DTnc 